\title{
The relationship between pulmonary function metrics and radiation-induced lung injury
}

\author{
J ustin M. Linam ${ }^{1}$, David Madtes ${ }^{2}$, Laura Chow ${ }^{3}$, Karen Liu ${ }^{4}$, Rodney Schmidt $^{5}$, Shilpen Patel ${ }^{6}$ \\ 1. University of Washington Medical School, Seattle, WA, USA. 2. Department of Pulmonary and Critical Care Medicine, \\ University of Washington, Seattle, WA, USA. 3. Department of Medicine, University of Washington Medical Center, Seattle, \\ WA, USA. 4. Department of Biostatistics, Fred Hutchinson Cancer Research Center, Seattle, WA, USA. 5. Department of \\ Pathology, University of Washington, Seattle, WA, USA. 6. Department of Radiation Oncology, University of Washington, \\ Seattle, WA, USA
}

Correspondence: Justin M. Linam. Address: University of Washington Medical School, Seattle, WA 98112, USA.

Email: jlinam@uw.edu

Received: August 9, 2012

Accepted: November 20, 2012

Online Published: December 10, 2012

DOI : $10.5430 /$ jst.v3n1p6

URL: http://dx.doi.org/10.5430/jst.v3n1p6

\section{Abstract}

Introduction: Radiation-induced lung injury (RILI) is a potentially fatal yet incompletely understood complication following radiation therapy. Models have been posited to predict RILI risk but have produced inconsistent results. Here we evaluate pulmonary function testing (PFT) metrics vs. RILI incidence with the hope of establishing clinical thresholds for RILI risk.

Methods: Our study population consisted of adult patients who completed conventionally-fractionated, definitive external beam RT for non-small cell lung cancer in a five-year period (January 1, 2006 to December 31, 2010). All patients were treated by a single radiation oncologist with identical technique, with dose prescriptions ranging from 50.4-74.4 Gray. We collected demographic and treatment variables, pre-radiation PFT values, and $\geqslant$ grade III RILI events.

Results: There were 62 patients in our dataset. RILI occurred in 6 patients. No significant associations were seen between age, radiation dose, and the use of chemotherapy vs. the development of RILI. Tukey's plots were constructed for PFT parameters and showed no significant differences in PFT values (absolute or percent of predicted) among RILI vs. non-RILI patients.

Conclusions: Our study compared pulmonary function parameters and other variables versus the development of RILI in patients treated for non-small cell lung cancer by a single radiation oncologist. We found no correlation between PFT values and risk of RILI. More research is needed to understand better the risks of RILI and to develop a clinically-useful and consistent model for RILI prediction.

\section{Key words}

Lung cancer, Radiation, Radiation-induced lung injury, Radiation pneumonitis, Radiation therapy, Non-small cell lung cancer

\section{I ntroduction}

Radiation-induced lung injury (RILI) is a relatively common and potentially fatal complication resulting from radiation therapy to the lungs. The pathophysiology of RILI is incompletely understood but seems to occur due to a combination of 
direct cytotoxic as well as indirect, cytokine-mediated effects ${ }^{[1]}$. The risk of RILI varies with radiation treatment factors such as dose, volume of lung treated, and fractionation schedule, as well as with individual patient characteristics ${ }^{[2-4]}$.

Because RILI is the primary dose-limiting factor in the treatment of a variety of chest and thoracic malignancies, understanding and avoiding RILI is of great importance in radiation therapy. A number of predictive models have been posited to ascertain which patients are likely to develop RILI given similar radiation doses and treatment parameters ${ }^{[2-14]}$. Existing models have produced inconsistent results, however, and are often too complicated or extensive to be practicable clinically.

In the hopes of establishing simple and more reliable predictors of RILI risk, we undertook the present observational study. Here, we evaluate simple pulmonary function metrics versus the development of RILI in a population of patients treated for non-small cell lung cancer (NSCLC) to assess for thresholds below which the development of RILI is more likely.

\section{Materials and methods}

After receiving approval from the Institutional Review Board of the University of Washington, we consulted paper and electronic medical records, treatment and billing databases, tumor registries, and pathology records to compile our patient list. We included all patients from a five-year period (January 1, 2006 to December 31, 2010) treated definitively with external beam radiation for pathologically-confirmed NSCLC by a single radiation oncologist (SP) within our hospital system, which includes the University of Washington Medical Center, the Seattle Cancer Care Alliance, and Harborview Medical Center. We excluded patients under 18 years of age at the time of diagnosis; patients treated with hyper-fractionated regimens; patients who had received prior radiation to the mediastinum or lungs; patients who did not finish the prescribed treatment; patients without pulmonary function testing; and patients with less than 6 months of follow-up who did not develop radiation pneumonitis within the follow-up period.

We collected the following variables from the medical record: age at the time of treatment, tumor histology, and pulmonary function metrics before radiation treatment, radiation dose, radiation fractionation, and chemotherapeutic regimen. We searched the medical record for evidence of RILI and defined a RILI event as cough or dyspnea within 6 months of radiation therapy requiring steroids or supplemental oxygen, causing hospitalization, or resulting in death (Radiation Therapy Oncology Group [RTOG] grade 3, 4, or 5, respectively). We plotted the following absolute and "percent of predicted" values (i.e., the patient's score as a percent of the score predicted for someone of the same age, sex, height, and weight) before radiation therapy versus the incidence of RILI in our population: forced vital capacity (FVC), forced expiratory volume in one second (FEV1), the FEV1 to FVC ratio, diffusion capacity of the lung for carbon monoxide (DLCO), and the DLCO value after correcting for hemoglobin concentration (DLCO-adjusted).

\subsection{Treatment techniques}

All patients were treated with full four dimensional radiation treatments planning to allow for respiratory movement. All patients underwent a treatment planning CT scan of the chest, neck, and upper abdomen specifically for identification of tumor targets and normal anatomy. Specifically, the entire volume of the lungs was encompassed in the CT data set. Patients were immobilized in a vacuum-locked bag with the arms positioned above the head. The CT scan protocol was $2.5 \mathrm{~mm}$ through the lungs and tumor.

For all patients a gross tumor volume (GTV) was defined and contoured. It included the primary disease plus any involved regional lymph nodes as determined by size on CT to be $>1 \mathrm{~cm}$ and/or pathologically involved by mediastinoscopy. The clinical target volume (CTV) was defined and contoured with $7 \mathrm{~mm}$ around the GTV and contours around involved lymph node regions. A planning target volume (PTV) with a margin of 3-5 mm was placed around the CTV. Dose prescriptions were then made to the International Commission on Radiation Units (ICRU) reference points (isocenters) of the PTV. Prescriptions ranged from 50.4 - 73.4 Gy with conventional fractionation of 180-200 cGy per day. All patients had V20 
(lung volume receiving 20 Gy of radiation) under 35\%, and all patients' mean lung dose (MLD) was under 20 Gy. Although an attempt was made to cover the PTVs with 95\% of the prescription dose (corrected for heterogeneities), this was not always possible, resulting in varying minimum doses. Though Karnofsky Performance Score (KPS) was not routinely recorded in the medical record, it is our practice to offer radiation therapy only to patients with a KPS of 70 or higher.

We analyzed PFT values vs. dose prescription using Pearson's Correlation Coefficients to ascertain whether poorer PFT values might be associated with lower dose prescriptions, thereby confounding our primary analysis.

\subsection{Chemotherapy}

Forty patients (65\%) received chemotherapy as part of their definitive treatment. Of these patients, 21 (52\%) received platinum-based regimens, 17 (42\%) received taxane-based regimens, 2 (5\%) received etoposide alone, and 1 (2\%) received permetrexed alone.

\section{Results}

There were 333 patients treated for lung cancer by one radiation oncologist (SP), of which 284 were treated for NSCLC. After removing patients who were treated with palliative intent, patients without 6 months of follow-up, patients who failed to complete treatment, patients who had previously received radiation to the chest or thorax, and patients treated with hyper-fractionated or stereotactic body radiation therapy, there were 62 patients left in the dataset. These patients were treated definitively for NSCLC by a single radiation oncologist (SP) with external beam radiation and identical technique. Characteristics of this patient population are presented in Table 1. RILI occurred in 6 patients, all of which were grade 3. Demographic and treatment variables for RILI and non-RILI patients were analyzed using Chi-square or t-test and are presented in Table 2.

Table 1. Patient characteristics

\begin{tabular}{ll}
\hline No. of patients & 62 \\
Age (median \pm SD) & $39-87$ years $(69 \pm 10.4)$ \\
Gender & 37 males $(60 \%)$ \\
Dose range in cGy (mean \pm SD) & $5040-7440(6645 \pm 697)$ \\
Chemotherapy & 40 patients $(65 \%)$ \\
\hline
\end{tabular}

Pearson's Correlation Coefficients of PFT values vs. dose ranged from -0.124 (DLCO-adjusted) to +0.306 (FVC). This suggests there was no correlation between PFTs and radiation dose in our study population.

Table 2. Radiation-induced lung injury (RILI) patient characteristics.

\begin{tabular}{llll}
\hline & RILI (n=6) & No RILI (n=56) & p \\
\hline Age, mean in years \pm SD & $67.8 \pm 10.9$ & $66.8 \pm 10.5$ & n.s. \\
Received Chemo, number (\%) & $35(62 \%)$ & $5(83 \%)$ & n.s. \\
Received Platinum-based Chemo, number (\%) & $19(34 \%)$ & $2(33 \%)$ & n.s. \\
Radiation dose, mean in cGy \pm SD & $6666 \pm 897$ & $6440 \pm 679$ & n.s. \\
\hline
\end{tabular}

The box-plots (Tukey's plots) of the FEV1 to FVC ratio (FEV1/FVC) as well as the "percent of predicted" values for FEV1, FVC, and DLCO-adjusted are presented in Figures 1-4. Box-plots for absolute values for FEV1, FVC, DLCO, and 
DLCO-adjusted are available online as Supplemental Figures 1-5. There were no threshold values below which the development of RILI became more likely.

Figure 1. FEV1/FVC ratio vs. radiation-induced lung injury (RILI) incidence.

Two patients (one RILI and one non-RILI) did not have a pre-therapy FEV1/FVC ratio in the record. The dark line represents the median (RILI=69; no RILI=65), the box encloses the 25\%-75\% quartiles (RILI=69-72; no RILI=55-74). Range: RILI=63-79; no RILI=27-92. The dashed line shows 1.5 times the interquartile range (IQR); outlying values are shown with a circle.

Figure 2. Forced expiratory volume in 1 second (FEV1) "percent of predicted" vs. radiation-induced lung injury (RILI) incidence.

One patient who developed RILI did not have a pre-therapy FEV1 in the record. The dark line represents the median (RILI=70; no RILI=65), the box encloses the $25 \%-75 \%$ quartiles (RILI=70-98; no RILI=55-76.5). Range: RILI=69-107; no RILI=21-103). The dashed line shows 1.5 times the interquartile range (IQR); outlying value is shown with a circle.

Figure 3. Forced vital capacity (FVC) "percent predicted" vs. radiation-induced lung injury (RILI) incidence. One patient who did not develop RILI did not have a pre-therapy FVC in the record. The dark line represents the median (RILI=88; no RILI=86), the box encloses the $25 \%-75 \%$ quartiles (RILI=76.5-101; no RILI=70.5-93.5), and the dashed line shows the range (RILI=76-127; no RILI=39-111).
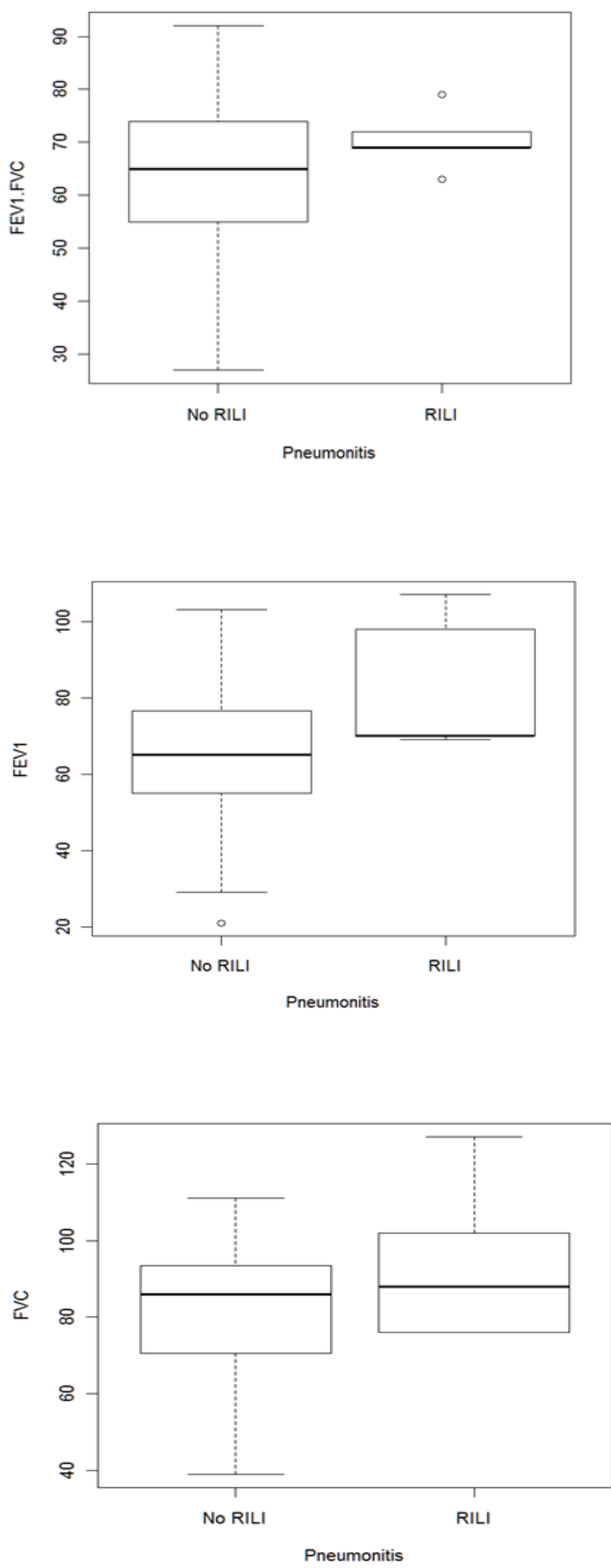
Figure 4. Diffusion capacity for carbon monoxide (DLCO) adjusted for hemoglobin "percent predicted" vs. radiation-induced lung injury (RILI) incidence.

Six patients (two RILI and four non-RILI) did not have "percent predicted” values for pre-therapy DLCO adjusted for hemoglobin in the record. The dark line represents the median (RILI=70.5; no RILI=53), the box encloses the $25 \%-75 \%$ quartiles (RILI=61.5-79.5; no RILI=39-64.5). Range: RILI=54-87; no RILI=21-108. The dashed line shows 1.5 times the interquartile range (IQR); outlying value is shown with a circle.

Figure 5. Diffusion capacity of carbon monoxide (DLCO) adjusted for hemoglobin (in $\mathrm{mL} \mathrm{CO} / \mathrm{min} / \mathrm{mm} \mathrm{Hg}$ ) vs. radiation-induced lung injury (RILI) incidence.

Seven patients (two RILI and five non-RILI) did not have absolute values for pre-therapy DLCO adjusted for hemoglobin in the record. The dark line represents the median (RILI=25.6; no RILI=15.5), the box encloses the $25 \%-75 \%$ quartiles (RILI=23.1-28.2; no RILI=11.1-18.2). Range: RILI=22.4-29.3; no RILI=6.7-29.0. The dashed line shows 1.5 times the interquartile range (IQR); outlying value is shown with a circle.
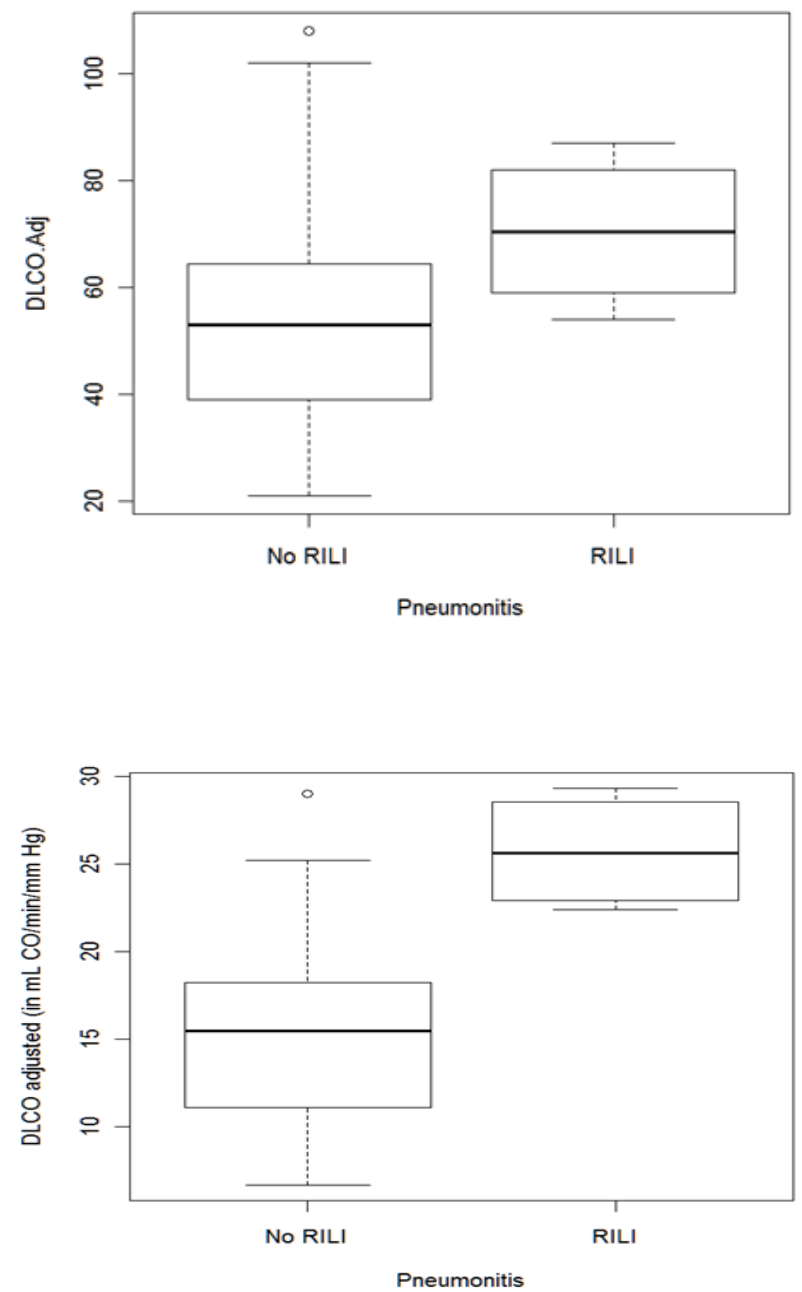

\section{Discussion}

Radiation-induced lung injury (RILI) is an important, dose-limiting cause of morbidity and mortality in radiation treatment of thoracic malignancies, particularly for non-small cell lung cancer (NSCLC). Although there can be radiologic abnormalities in RILI, the diagnosis is usually made clinically, based on dyspnea, non-productive cough, and physical exam findings (i.e., "fine crackles"). RILI grading and the decision to treat, however, depend mostly on the severity of the patient's cough and dyspnea and the degree to which these symptoms affect the activities of daily living. Unfortunately, cough and dyspnea also result from lung cancer itself, pre-existing pulmonary diseases that are often comorbid with lung cancer (COPD, e.g.), and other medical conditions (CHF, e.g.). This makes the definitive diagnosis of RILI difficult in many situations and explains the broad range of reported rates of RILI, which vary from $5 \%$ to over $30 \%$ of patients treated for NCSLC ${ }^{[14,15]}$.

The pathophysiology of RILI continues to be studied, but it is clear that ionizing radiation causes direct cell death, triggers apoptotic pathways, and precipitates the release of a number of cytokines, all of which result in the common endpoint of pulmonary fibrosis ${ }^{[15-17]}$. We also know that the risk of RILI increases with increasing radiation dose; whether the risk is best correlated with mean lung dose or with V20 or V30 (the volume of normal lung that receives more than 20 or 30 Gy, respectively) is debated in the literature ${ }^{[5,12,18-19]}$. For example, Graham et al. proposed using the V20 a patient's dose-volume histogram to predict RILI development ${ }^{[19]}$. Although this model seemed to work well in the original patient 
cohort, a recent study using the model with the Radiation Therapy Oncology Group (RTOG) 9311 trial patients found that the model did not fit well with this cohort ${ }^{[20]}$. These authors instead proposed a simple, clinically-useful nomogram using mean lung dose (MLD) and the superior-inferior position of the dose center to predict the risk of RILI.

Despite the correlation of radiotherapeutic parameters with RILI incidence, some patients are at higher risk for RILI than others, even with identical treatment parameters and plans. This has led some investigators to focus on patient characteristics and RILI incidence to better understand these individual susceptibilities. In fact, a recent study of 438 patients treated for NSCLC found patient characteristics such as age, performance status, smoking status, and FEV1 to be more predictive for RILI development than dosimetric parameters ${ }^{[13]}$. Videtic et al. found low DLCO ( $<60 \%$ predicted) to be significantly associated with more treatment breaks and worse survival in a study of patients treated with radiation therapy for small-cell lung cancer ${ }^{[21]}$. Other studies have also identified Karnofsky performance status, FEV1, and a diagnosis of COPD as significant factors in predicting RILI risk and other treatment-related complications ${ }^{[8,22]}$.

The importance of these clinical factors have led to the development of complex neural network modeling algorithms that incorporate dosimetric as well as clinical parameters in order to most accurately predict RILI risk ${ }^{[9-11]}$, but these models seem too complicated and unwieldy to be clinically useful or practicable for most radiation oncologists. A recent study proposes a new metric, TFS5, which combines the volume of lung spared from receiving more than 5 Gy and the transfer coefficient for carbon monoxide (KCO) ${ }^{[14]}$. The predictive value of this metric is promising in their study, but whether the results can be generalized to other institutions and patient populations remains to be seen.

Because of the discrepancies in the literature regarding the best predictors of RILI and the complexity of many of the extant predictive models, we undertook the current study in an attempt to find a simple, readily available threshold of lung function below which the risk of RILI would increase significantly. Even though it is pathophysiologically related to damage on the cellular and subcellular level, the diagnosis of RILI is made clinically. Thus, a patient's pulmonary function and respiratory reserve seem to dictate whether RILI will become clinically significant and how well a given patient will tolerate radiation damage to healthy lung tissue. This principle motivated the present study.

In assessing the pulmonary function parameters versus the development of RILI, however, we found similar (and often, higher) pre-therapy PFTs in patients who developed RILI compared to patients who did not. We also failed to find a threshold of pulmonary function below which RILI development was more likely. This is discrepant with other studies, which have found an association between poor FEV1 and RILI. Clearly, more research is needed to parse out which patient characteristics affect RILI development and how they interact with treatment parameters. The search for a simple, clinically-useful, and generalizable RILI prediction model continues.

\section{Conclusion}

Our study compared pulmonary function parameters versus the development of radiation-induced lung injury (RILI) in patients treated with definitive radiation therapy for non-small cell lung cancer by a single radiation oncologist within a single hospital system. No clinically-useful thresholds for lung function parameters were found that would allow the clinician to accurately predict the development of RILI.

\section{Conflict of interests}

We have no conflict of interests to report as regards this research, nor do we have any financial disclosures to make.

\section{References}

[1] Morgan GW, Breit SN. Radiation and the lung: a reevaluation of the mechanisms mediating pulmonary injury. Int J Radiat Oncol Biol Phys. 1995; 31(2): 361. http://dx.doi.org/10.1016/0360-3016(94)00477-3 
[2] Marks LB, Bentzen SM, Deasy JO et al. Radiation dose-volume effects in the lung. Int J Radiat Oncol Biol Phys. 2010 Mar 1; 76(3 Suppl): S70-6. Review. PMid:20171521 http://dx.doi.org/10.1016/j.ijrobp.2009.06.091

[3] Yorke ED, Jackson A, Rosenzweig KE et al. Correlation of dosimetric factors and radiation pneumonitis for non-small-cell lung cancer patients in a recently completed dose escalation study. Int J Radiat Oncol Biol Phys. 2005; 63: 672-682. PMid:15939548 http://dx.doi.org/10.1016/j.ijrobp.2005.03.026

[4] Hernando ML, Marks LB, Bentel GC et al. Radiation-induced pulmonary toxicity: A dose-volume histogram analysis in 201 patients with lung cancer. Int J Radiat Oncol Biol Phys. 2001; 51: 650-659. http://dx.doi.org/10.1016/S0360-3016(01)01685-6

[5] Kwa SL, Lebesque JV, Theuws JC et al. Radiation pneumonitis as a function of mean lung dose: An analysis of pooled data of 540 patients. Int J Radiat Oncol Biol Phys. 1998; 42: 1-9. http://dx.doi.org/10.1016/S0360-3016(98)00196-5

[6] Graham MV, Purdy JA, Emami B et al. Clinical dose-volume histogram analysis for pneumonitis after 3D treatment for non-small cell lung cancer (NSCLC). Int J Radiat Oncol Biol Phys. 1999; 45: 323-329. http://dx.doi.org/10.1016/S0360-3016(99)00183-2

[7] Seppenwoolde Y, Lebesque JV, de Jaeger K et al. Comparing different NTCP models that predict the incidence of radiation pneumonitis. Normal tissue complication probability. Int J Radiat Oncol Biol Phys. 2003 Mar 1; 55(3): 724-35. http://dx.doi.org/10.1016/S0360-3016(02)03986-X

[8] Moreno M, Aristu J, Ramos LI et al. Predictive factors for radiation-induced pulmonary toxicity after three-dimensional conformal chemoradiation in locally advanced non-small-cell lung cancer. Clin Transl Oncol. 2007 Sep; 9(9): 596-602. PMid:17921108 http://dx.doi.org/10.1007/s12094-007-0109-1

[9] Chen S, Zhou S, Zhang J et al. A neural network model to predict lung radiation-induced pneumonitis. Med Phys. 2007 Sep; 34(9): 3420-7. PMid:17926943 http://dx.doi.org/10.1118/1.2759601

[10] Das SK, Chen S, Deasy JO et al. Combining multiple models to generate consensus: application to radiation-induced pneumonitis prediction. Med Phys. 2008 Nov; 35(11): 5098-109. PMid:19070244 http://dx.doi.org/10.1118/1.2996012

[11] Su M, Miften M, Whiddon C et al. An artificial neural network for predicting the incidence of radiation pneumonitis. Med Phys. 2005 Feb; 32(2): 318-25. PMid:15789575 http://dx.doi.org/10.1118/1.1835611

[12] Fay M, Tan A, Fisher R et al. Dose-volume histogram analysis as predictor of radiation pneumonitis in primary lung cancer patients treated with radiotherapy. Int J Radiat Oncol Biol Phys. 2005 Apr 1; 61(5): 1355-63. PMid:15817337 http://dx.doi.org/10.1016/j.ijrobp.2004.08.025

[13] Dehing-Oberije C, De Ruysscher D, van Baardwijk A et al. The importance of patient characteristics for the prediction of radiation-induced lung toxicity. Radiother Oncol. 2009 Jun; 91(3): 421-6. Epub 2009 Jan 13. PMid:19147245 http://dx.doi.org/10.1016/j.radonc.2008.12.002

[14] Jenkins P, Watts J. An improved model for predicting radiation pneumonitis incorporating clinical and dosimetric variables. Int J Radiat Oncol Biol Phys. 2011 Jul 15; 80(4): 1023-9. Epub 2011 May 3. PMid:21543165 http://dx.doi.org/10.1016/j.ijrobp.2010.03.058

[15] McDonald S, Rubin P, Phillips TL, Marks LB. Injury to the lung from cancer therapy: clinical syndromes, measurable endpoints, and potential scoring systems. Int J Radiat Oncol Biol Phys. 1995 Mar 30; 31(5): 1187-203.

http://dx.doi.org/10.1016/0360-3016(94)00429-O

[16] Graves PR, Siddiqui F, Anscher MS, Movsas B. Radiation pulmonary toxicity: from mechanisms to management. Semin Radiat Oncol. 2010 Jul; 20(3): 201-7. PMid:20685583 http://dx.doi.org/10.1016/j.semradonc.2010.01.010

[17] Rubin P, Johnston CJ, Williams JP et al. A perpetual cascade of cytokines postirradiation leads to pulmonary fibrosis. Int J Radiat Oncol Biol Phys. 1995; 33: 99. http://dx.doi.org/10.1016/0360-3016(95)00095-G

[18] Kim TH, Cho KH, Pyo HR et al. Dose-volumetric parameters for predicting severe radiation pneumonitis after three-dimensional conformal radiation therapy for lung cancer. Radiology. 2005; 235(1): 208. PMid:15703313 http://dx.doi.org/10.1148/radiol.2351040248

[19] Graham MV, Purdy JA, Emami B et al. Clinical dose-volume histogram analysis for pneumonitis after 3D treatment for non-small cell lung cancer (NSCLC). Int J Radiat Oncol Biol Phys. 1999 Sep 1; 45(2): 323-9. http://dx.doi.org/10.1016/S0360-3016(99)00183-2

[20] Bradley JD, Hope A, El Naqa IE et al. A nomogram to predict radiation pneumonitis, derived from a combined analysis of RTOG 9311 and institutional data. Int J Radiat Oncol Biol Phys. 2007 Nov 15; 69(4): 985-92. Epub 2007 Aug 6. PMid:17689035 http://dx.doi.org/10.1016/j.ijrobp.2007.04.077

[21] Videtic GM, Stitt LW, Ash RB et al. Impaired diffusion capacity predicts for decreased treatment tolerance and survival in limited stage small cell lung cancer patients treated with concurrent chemoradiation. Lung Cancer. 2004 Feb; 43(2): 159-66.

PMid:14739036 http://dx.doi.org/10.1016/j.lungcan.2003.08.026 
[22] Robnett TJ, Machtay M, Vines EF et al. Factors predicting severe radiation pneumonitis in patients receiving definitive chemoradiation for lung cancer. Int J Radiat Oncol Biol Phys. 2000 Aug 1; 48(1): 89-94. http://dx.doi.org/10.1016/S0360-3016(00)00648-9 